\title{
The Social Marginal Cost Curve and a Corner Solution of the Second-Best Level of Public Good Provision: A Review and an Extension
}

\author{
Ming Chung Chang ${ }^{a}$, Hsiao-Ping Peng ${ }^{b}$, and Yan-Ching $\mathrm{Ho}^{c}$
}

JEL-Classification: H21, H41.

Keywords: second-best public good provision, social marginal cost, demand-shift effect, weak Laffer effect.

\section{SUMMARY}

Assume that the private goods and the public good are weakly separable, the private goods are gross complements, and the private utility function is a homogeneous of degree one function with constant elasticity of substitution. We demonstrate that, under commodity taxation, the social marginal cost curve of public good provision is initially upward sloping and eventually becomes downward sloping. Moreover, the social marginal cost eventually falls below the private marginal cost. These unusual properties arise from a demand-shift effect: An increase in the tax rate raises the marginal willingness to pay for the public good since it pushes up the unit cost of private utility, hence making the public good more attractive than private goods. In other words, the supply of the public good creates its own demand when the funding to cover production costs is raised through distortionary commodity taxes. It follows that there may exist three solutions to the first-order condition for the second-best problem: two of them are interior solutions and one is a corner solution.

a Professor, Graduate Institute of Industrial Economics, National Central University, No. 300, Chung-Ta Road, Chung-Li City 32001, Taiwan, Email: mcchang@mgt.ncu.edu.tw, Tel.: 8863-4227617, Fax: 886-3-4226134.

b Associate Professor, Yu Da University, Taiwan, Email: cindy@ydu.edu.tw.

c Ph.D. candidate, Graduate Institute of Industrial Economics, National Central University, Taiwan, Email: r4688122@hotmail.com. 


\section{Introduction}

This paper studies the optimal provision level of a public good financed by a consumption tax in an economy with a single consumer (who might represent a population of identical agents). Pigou (1947, p. 34) claims that when the funding to cover production costs is raised through distortionary taxes, the social marginal cost of the public good is higher than its private marginal cost (Rule Property), and hence the second-best level of public good provision is lower than the first-best level (Level Property). Despite being a long-standing and central issue in public economics, it appears to be an unresolved one (SLEmrod and Yitzhaki, 2001, p. 189; Christiansen, 2007, p. 25). For example, regarding the level property, the existing literature does not confirm Pigou's conjecture, except for two special cases (Chang and Peng, 2012, p. 651).

We assume that the first-best regime is well-behaved, i.e., the demand curve for the public good (the marginal willingness to pay for the public good as a function of the level of public good provision) is downward sloping, and it meets the private-marginal-cost (PMC) curve at a unique interior point. Therefore, if the second-best regime is abnormal, then the abnormity does not arise from unusual assumptions.

Consider the second-best regime. We can divide the net effect of an increment in the level of public good provision into benefits and costs in many ways, ${ }^{1}$ but we shall assume that if there are indirect effects, then all of them are included in the cost side. In other words, we assume that the benefit side does not change (i.e., the demand curve for the public good does not shift) when the commodity taxation substitutes for the lump-sum taxation and instead concentrate on the value to which this should be equated (or the cost measure).

The literature implicitly assumes that there exists a unique solution to the firstorder condition for the second-best problem, and that the solution is an interior one. Our goal is to verify this assumption. To this end, we begin with characterizing the social-marginal-cost (SMC) curve. $^{2}$ We demonstrate that the SMC curve is initially upward sloping and eventually becomes downward sloping. Moreover, the SMC curve eventually falls below the PMC curve. These unusual

1 This explains why DAнLby $(2008$, p. 2) notes that "While a substantial literature on the MCF has developed over the last twenty years, much of this literature is fragmented because authors have used different measures for the MCF" where the MCF is the SMC divided by PMC.

2 Atrinson and Stern (1974) as well as Gronberg and Liv (2001) already use the demand and supply curves for the public good to illustrate their arguments. However, they do not formally examine these two curves. 
properties arise from a demand-shift effect, which states that an increase in the tax rate raises the marginal willingness to pay for the public good since it pushes up the unit cost of private utility, hence making the public good be more attractive relative to the private goods. In other words, the supply of the public good creates its own demand when the funding to cover production costs is raised through distortionary commodity taxes! It follows that there may exist three solutions to the first-order condition for the second-best problem: two of them are interior and one is corner.

Section 2 sets up the model. Because the SMC is the PMC plus the marginal deadweight loss (MDL), in Section 3, we decompose the MDL into four effects. Moreover, we will compare the four effects. We follow Chang and Peng (2012, p. 651) to emphasize the distinction between a case with gross substitution, where the private goods are gross substitutes, and a case with gross complementarity, where the private goods are gross complements. This is because Gaube (2000) demonstrates that if the public good and the private goods are weakly normal, then in the gross substitution case the level property holds. In contrast, in the gross complementarity case, there exists a numerical example in which the level property reverses. The results obtained in Section 3 are used tom predict the results of Sections 4 and 5.

In Sections 4 and 5 we will conduct the main analysis. To this end, we restrict our attention to the model of Wilson (1991a), namely, our Example 3.2, where there are one untaxed private good, one taxed private good, and one public good, the Marshallian demand for each private commodity is independent of the public good provision level (i.e., the private goods and the public good are weakly separable), and the private utility function is a homogeneous of degree one CES (i.e., constant elasticity of substitution) function. In Section 4, we address the two issues regarding the SMC curve mentioned above. In particular, it is demonstrated that the SMC curve may become downward sloping and it may lie below the PMC curve when the level of public good provision is high enough. In Section 5, we study the three fundamental issues mentioned above, and demonstrate that there may exist three solutions to the first-order condition for the second-best problem, and the second-best solution may be a corner one. At the end of this section, we discuss whether the assumptions made in this paper are reasonable or not. Section 6 concludes this paper. 


\section{The Model}

There is a single consumer whose utility depends on $x, \omega+z$, and $g$, where $x$ is one taxed private commodity; $\omega$ is the time endowment; $z$ is the "negative labor supply"; and $g$ is one public good. The pre-tax price of $x$ is $p$, which is assumed to be constant; the post-tax price of $x$ is $q$. It is assumed that $z$ is not taxed, and hence its price is normalized to be 1 , a standard assumption in the literature (Atkinson and Stiglitz, 1980, p.371-2; Stern, 1986, p. 298). The public good has a positive and constant marginal cost $c$.

We define notation as follows:

$E(q, g, u) \equiv$ expenditure function where $u$ is the utility level.

$H(q, g, u) \equiv$ Hicksian demand function for $x$.

Any subscript denotes partial differentiation, e.g., $E_{g}$ stands for $\partial E / \partial g$.

$W(q, g, u) \equiv-E_{g}(q, g, u)$, i.e., it is the marginal willingness to pay (MWP) for the public good.

$V(q, g, \omega+y) \equiv$ indirect utility function where $y$ is the negative lump-sum tax.

$X(q, g, \omega+y) \equiv$ Marshallian demand function for $x$.

$t \equiv q-p$.

$R(q, g, \omega+y) \equiv t X(q, g, \omega+y)$, i.e., it is the commodity tax revenue function.

Here, $g^{F}, w^{F}$, and $u^{F}$ are the first-best levels for $g, W$, and $u$, respectively, and $g^{S}$, $w^{S}, u^{S}$, and $q^{S}$ are the second-best levels for $g, W, u$, and $q$, respectively.

Since $y$ is the negative lump-sum tax, the first-best program is:

$$
\max _{g} V(p, g, \omega+y) \text { s.t. } y=-c g .
$$

In contrast, the second-best program is:

$$
\max _{q, g} V(q, g, \omega) \text { s.t. } R(q, g, \omega)=c g,
$$

because $R(q, g, \omega)$ is the commodity tax revenue.

In order to guarantee that the first-best regime is well-behaved, the following two assumptions are made:

A1: The public good is a normal good with declining marginal benefit. That is, $W_{u} \geq 0$ and $W_{g}<0$.

A2: There exists a unique first-best solution. Moreover, it is an interior solution, i.e., $0<g^{F}<\omega / c$.

3 Since $0 \leq g \leq \omega / c$, we refer to $g$ as an interior solution if and only if $g \in(0, \omega / c)$. 


\section{The Decomposition of MDL}

In Section 3.1, we decompose the MDL into four effects pointed out in the literature. In Section 3.2, the four effects are compared.

\subsection{The Four Effects}

The first-best $g$ satisfies the following equation:

$$
W(p, g, u)=c .
$$

In the first-best regime, the funding to cover production costs for the public good is financed by the lump-sum tax (i.e., $y=-c g)$, and hence $u=V(p, g, \omega-c g)$. We will let $u^{F}(g)$ denote $V(p, g, \omega-c g)$. Therefore, the first-best $g$ is determined by the following equation:

$$
W\left(p, g, u^{F}(g)\right)=c .
$$

We next consider the second-best regime. We divide the net effect of an increment in the level of public good provision into benefits and costs in many ways, but we shall assume that the benefit side is represented by $W\left(p, g, u^{F}(g)\right)$ and concentrate on the value to which this should be equated (or the cost measure). This means that if there are indirect effects, then all of them should be included in the cost measure.

According to Eq. (36) of KING (1986, p. 282), the second-best $g$ satisfies the following equation:

$$
W(q, g, u)=c+\left(1-\frac{\mu}{\lambda}\right) W(q, g, u)-t H_{g}(q, g, u),
$$

where $\lambda$ is the Lagrange multiplier of the government's budget constraint, and $\mu \equiv V_{y}+\lambda R_{y}$ is the so-called "social marginal utility of income" (Diamond, 1975, p.338). According to (7) of Diamond (1975), $1-\mu / \lambda$ equals $-t H_{q} / H$, implying that (3.3) can be rewritten as:

$$
W(q, g, u)=c-\frac{t H_{q}(q, g, u)}{H(q, g, u)} W(q, g, u)-t H_{g}(q, g, u) .
$$


This standard formula reveals two famous effects. First, the second term of the right-hand side is non-negative, ${ }^{4}$ and it represents the Pigou effect, the distortionary effect with which Pigou is concerned. Since this effect is well known, we need not explain it. Second, the third term of the right-hand side is referred to by Batina (1990, p. 129-30) as the provision effect, because this term captures "the indirect effect of providing the public good on the tax revenue". In general, this effect may go either way depending upon the sign of $H_{g}$. For example, if the public good is a Hicksian substitute to the taxed good (i.e., $H_{g}$ is negative), then the provision effect is positive. We can use the argument made by ATKINSON and STERN (1974, p. 122) to explain this result: If increased government expenditure leads to a smaller consumption of taxed private good, this raises the revenue which has to be raised and hence increases the cost measure.

As shown above, Eq. (36) of KING (1986) can be rewritten into Eq. (3.4), which captures the Pigou effect and the provision effect. However, this equation misses the following two effects: the "income effect" pointed out by Wilson (1991b, p. 158), and the "demand-shift" effect emphasized by De Bartolome (1996; 1997) and $N_{G}$ (2000). In order to use a single equation to capture all the effects, we continue to rewrite Eq. (3.4). Since

$$
W(q, g, u)=W(p, g, u)+[W(q, g, u)-W(p, g, u)],
$$

(3.4) can be rewritten as:

$$
W(p, g, u)=c-\frac{t H_{q}(q, g, u)}{H(q, g, u)} W(q, g, u)-t H_{g}(q, g, u)-\Delta W(q, g, u),
$$

where $\Delta W(q, g, u) \equiv W(q, g, u)-W(p, g, u)$. We may refer to the fourth term of the right-hand side as the "demand-shift" effect since $W$ represents the "demand price" for the public good. According to (3) of Chang (2000), $W_{q}=-H_{g}$. Therefore, the demand-shift effect is a dual counterpart of the provision effect, and it works against the provision effect. For example, if the public good is a Hicksian

4 It is known that $E$ is concave with respect to $q$. It follows that $H_{q} \leq 0$. Therefore, from $t>0$ it follows that $-t H_{q} W / H \geq 0(t>0$, as long as $g>0)$.

5 Actually, Diamond and Mirrlees (1971, p. 271) and Atrinson and Stern (1974, p. 122) already emphasize the uncompensated counterpart of the provision effect, namely, $-t X_{g}$. We follow Batina and Ihori (2005, p. 34) to call $-t H_{g}$ the "compensated provision effect" if we want to distinguish it from $-t X_{g}$. 
substitute to the taxed good, then, as indicated by $\Delta W>0,{ }^{6}$ the commodity tax raises the MWP, and hence reduces the cost measure (as mentioned in the introduction, we eventually let each indirect effect be included in the cost side). To our knowledge, only DE BARTOLOME (1996; 1997, p. 286) and NG (2000, p. 256) mention this demand-shift effect. ${ }^{7}$

In the second-best regime, $y=0$, and hence $u=V\left(q^{S}(g), g, \omega\right)$, where $q^{S}(g)$ stands for the post-tax price that affords revenue $c g$ to the government, i.e., $q^{S}(g)$ solves the following budget constraint:

$$
R(q, g, \omega)=c g
$$

Let $u^{S}(g)$ denote $V\left(q^{S}(g), g, \omega\right)$. Equation $\left(3.4^{\prime}\right)$ implies that the second-best $g$ is determined by the following equation:

$$
W\left(p, g, u^{S}(g)\right)=c+\Phi\left(q^{S}(g), g, u^{S}(g)\right)
$$

where $\Phi$ represents the marginal deadweight loss:

$$
\Phi(q, g, u) \equiv-\frac{t H_{q}(q, g, u)}{H(q, g, u)} W(q, g, u)-t H_{g}(q, g, u)-\Delta W(q, g, u) .
$$

Since $W\left(p, g, u^{S}(g)\right)=W\left(p, g, u^{F}(g)\right)+\left[W\left(p, g, u^{S}(g)\right)-W\left(p, g, u^{F}(g)\right)\right]$, Eq. (3.6) can be rewritten as the following equation:

$$
\begin{aligned}
W\left(p, g, u^{F}(g)\right)= & c+\Phi\left(q^{S}(g), g, u^{S}(g)\right) \\
& +\left[W\left(p, g, u^{F}(g)\right)-W\left(p, g, u^{S}(g)\right)\right]
\end{aligned}
$$

Note that $W\left(p, g, u^{S}(g)\right)-W\left(p, g, u^{F}(g)\right)$ represents the income effect pointed out by Wilson (1991, p. 158). Since

$$
u^{F}(g) \geq u^{S}(g) \forall g
$$

6 If $H_{g}<0$, then $W_{q}>0$, implying that $\Delta W>0$.

7 The first author is indebted to Professor Yew-Kuang Ng. In presenting one of his working papers regarding the optimal size of public spending in a 1997 seminar at the Department of Economics, National Taiwan University, Professor Ng pointed out that a working paper version of CHANG (2000) means that there is a counteracting effect to the compensated provision effect, and this counteracting effect shifts the demand curve for the public good. 
from A1 it follows that the income effect is non-negative. This effect means that, as indicated by the above equation, the distortionary commodity tax unambiguously reduces the real income of the consumer, and hence decreases the MWP (i.e., increases the cost measure).

In summary, comparing $\left(3.6^{\prime}\right)$ with (3.2) reveals that the right-hand side of $\left(3.6^{\prime}\right)$ represents the SMC of the commodity taxation, and hence

$$
\Phi\left(q^{S}(g), g, u^{S}(g)\right)+\left[W\left(p, g, u^{F}(g)\right)-W\left(p, g, u^{S}(g)\right)\right],
$$

which consists of the four effects mentioned above, represents the MDL of the commodity taxation. Either one of the Pigou effect and the income effect is non-negative. In contrast, the sign of either one of the provision effect and the demand-shift effect is determined by the sign of $H_{g}$. Moreover, the provision effect and the demand-shift effect have opposite signs.

\section{The Four Effects for Two Famous Approaches}

There are two famous approaches in the public finance literature, as noted by Ballard and Fullerton (1992, p. 124) and Chang (2000, p. 86). One assumes that the private goods and the public good are weakly separable. In this approach, if $x$ is normal, then $H_{g} \leq 0,{ }^{8}$ and hence the provision effect is non-negative. The other approach assumes that the Hicksian demand for each taxed commodity does not depend upon the level of public good provision, i.e., $H_{g}=0$. In this approach, the provision effect vanishes. In summary, in the above two approaches, the provision effect is non-negative, and hence the demand-shift is non-positive.

Chang (2000) has already established that, in the second approach, the level property unambiguously holds. Accordingly, hereafter, we restrict our attention to the first approach. In order to take a closer look at the four effects, let us present the following famous example:

Example 3.1. The total utility function of the consumer is $U(x, \omega+z)+f(g)$, and the private utility function $U$ is homogeneous of degree 1 with respect to $x$ and $\omega+z$.

8 It is clear that the Marshallian demand for each taxed commodity does not depend upon the level of public good provision, i.e., $X_{g}=0$. Drawing upon KING (1986, Eq. (11)) yields the following Slutsky equation: $X_{g}=H_{g}+W X_{y}$. Accordingly, if $X_{g}=0$ and $X_{y} \geq 0$, then $H_{g} \leq 0$.

9 In Example 1 of Wilson (1991b), there is more than one consumer, and they may differ with respect to endowments. When the consumers have the same endowment, Example 1 of WiLson (1991b) degenerates into our Example 3.1. 
We let $e(q)$ stand for consumer expenditures per unit of private utility at price $q$ :

$$
e(q) \equiv \min _{x, z} q x+(\omega+z) \text { s.t. } U(x, \omega+z)=1 .
$$

Since $u=U(x, \omega+z)+f(g)$, the private utility needed to achieve total utility $u$ under public good supply $g$ is $u-f(g)$, and hence $E(q, g, u)=[u-f(g)] e(q)$. From $H=E_{q}$ and $W=-E_{g}$, it follows that:

$$
\begin{aligned}
H(q, g, u) & =[u-f(g)] h(q), \\
W(q, g) & =f^{\prime}(g) e(q),
\end{aligned}
$$

where $h(q) \equiv e^{\prime}(q)$. Because $e(q)$ is consumer expenditures per unit of private utility at price $q$, the level of private utility is $(\omega+y) / e(q)$. It follows that:

$$
\begin{aligned}
X(q, \omega+y) & =h(q)(\omega+y) / e(q), \\
R(q, \omega+y) & =t h(q)(\omega+y) / e(q) .
\end{aligned}
$$

According to (3.10), $H_{g}=-f^{\prime}(g) h(q)<0$, and hence the provision effect is positive. It follows that the demand-shift effect is negative. As shown in (3.11), $W$ does not depend on $u$, implying that the income effect does not exist.

We are in a position to further interpret the demand-shift effect. Equation (3.11) indicates that, in Example 3.1, $W$ is proportional to the unit cost of private utility. Therefore, an increase in the tax rate raises the MWP for the public good, because it pushes up the unit cost of private utility and hence make the public good be more attractive, relative to the private goods. ${ }^{10}$ In other words, the supply of the public good creates its own demand when the funding to cover production costs is raised through distortionary taxes!

\subsection{The Four Effects Compared}

This subsection aims at comparing the four effects for Example 3.1. First, we compare the compensated provision effect with the demand-shift effect. Second, we compare the Pigou effect with the compensated provision effect. [As mentioned in Section 3.1, for Example 3.1, the income effect does not exist.]

10 The reasoning of DE BARTOLOME (1996) is as follows. The commodity taxation causes the household to consume more untaxed numeraire, thereby lowering the marginal utility associated with the numeraire. The marginal benefit of the public good thus rises. 
According to our knowledge, these two comparisons have not been conducted in the previous literature.

\section{The Compensated Provision Effect and the Demand-Shift Effect Compared}

We next draw on a result obtained by Wilson (1991b) to demonstrate that the provision effect is dominated by the demand-shift effect. One feature of Wilson's approach is utilizing the following "loss function" to analyze the level problem:

$$
L(q, g, u) \equiv q[H(q, g, u)-H(p, g, u)]+Z^{C}(q, g, u)-Z^{C}(p, g, u),
$$

where $Z^{C}(q, g, u)$ is the compensated demand function for $z$. The above equation can be rewritten as:

$$
L(q, g, u)=[E(q, g, u)-E(p, g, u)]-t H(q, g, u),
$$

which is the so-called "Kay-Pazner-Sadka excess burden measure" (TRIEST, 1990, p. 558-9, Eq. (1)). ${ }^{11}$ Partially differentiating $3.14^{\prime}$ with respect to $q$ and $g$ yields:

$$
\begin{gathered}
L_{q}(q, g, u)=-t H_{q}(q, g, u), \\
L_{g}(q, g, u)=-t H_{g}(q, g, u)-[W(q, g, u)-W(p, g, u)] .
\end{gathered}
$$

Therefore:

$$
\Phi(q, g, u)=\frac{W(q, g, u)}{H(q, g, u)} L_{q}(q, g, u)+L_{g}(q, g, u) .
$$

Equations (3.15) and (3.16) indicate that $L_{q}$ represents the Pigou effect, and $L_{g}$ represents the sum of the provision and demand-shift effects. As mentioned, for Example 3.1, $H_{g}<0$, implying that the provision effect is positive (i.e., $-t H_{g}>0$ ), whereas the demand-shift effect is negative (i.e., $-\Delta W<0$ ). According to (24) of Wilson (1991b), for Example 3.1: ${ }^{12}$

11 Gronberg and Liv (2001, p. 436) utilize this excess burden measure to formulate the secondbest problem.

12 As mentioned, Example 3.1 is the representative-consumer version of Example 1 of WILson (1991b). Therefore, we can apply (24) to our Example 3.1. 


$$
L_{g}(q, g, u)=-f^{\prime}(g) u-f(g) L(q, g, u)<0,
$$

implying that the provision effect is dominated by the demand-shift effect.

We now explain why $L_{g}<0$. Wilson (1991b, p. 159) notes that if the private goods are weakly separable from the public good, then one can define the concept of "private utility." Given commodity tax rates and "total utility" held constant, a rise in the public good provision affects the deadweight loss only by reducing private utility. In other words, a higher public good provision may be viewed as representing "a transfer of resources away from the distorted private sector and into the government-controlled sector. With fewer resources employed in the sector that misallocates them, the deadweight loss should be lower."

\section{The Pigou Effect and the Compensated Provision Effect Compared}

It is next to compare the Pigou effect with the provision effect. To this end, let us follow Wilson (1991a) and Gaube (2000, Section 5) to restrict our attention to the following example, which is a special case of Example 3.1.

Example 3.2. The total utility function of the consumer is $U(x, \omega+z)+f(g)$, and the private utility function $U$ is homogeneous of degree 1 with respect to $x$ and $\omega+z$. Moreover, $\sigma$ is constant where $\sigma$ denotes the elasticity of substitution between $x$ and $\omega+z$.

In Example 3.2 the private utility function $U$ possesses the following closed form:

$$
U(x, \omega+z)=a\left[b x^{\rho}+(1-b)(\omega+z)^{\rho}\right]^{1 / \rho},
$$

where $a, b$, and $\rho$ are constants, and $a>0,0<b<1$, and $\rho<1$. It is known that $\sigma=1 /(1-\rho)$.

According to (3.11), the MWP is $f^{\prime}(g) e(p)$. Note that the MWP does not depend on $u$, and hence the first-best $g$ can be found by simply solving (3.1), namely, $f^{\prime}(g) e(p)=c$. In order to guarantee that the two assumptions A1 and A2 are satisfied, we assume that:

$$
\begin{gathered}
f^{\prime \prime}(g)<0 \forall g \geq 0, \\
f^{\prime}(0) e(p)>c>f^{\prime}(\omega / c) e(p) .
\end{gathered}
$$

Equation (3.20a) implies that, in the (g,money) space, the MWP [i.e., $f^{\prime}(g) e(p)$ ] curve is downward sloping, whereas $(3.20 \mathrm{~b})$ guarantees that the MWP curve 
meets the PMC curve at a unique interior point. Moreover, for convenience of presentation, we assume that the public good is always desirable, i.e.:

$$
f^{\prime}(\omega / c)>0 .
$$

In Remark 4.1, we will show how to revise the results if (3.21) does not hold.

Equations (3.10) and (3.11), together with $\left(3.4^{\prime}\right)$, imply that the Pigou effect is represented by $-t e(q) h^{\prime}(q) f^{\prime}(g) / h(q)>0$; the provision effect is $t h(q) f^{\prime}(g)>0$; the demand-shift effect is $-[e(q)-e(p)] f^{\prime}(g)<0$. [As mentioned, the income effect does not exist.] In summary:

$$
\Phi(q, g)=f^{\prime}(g) \Gamma(q),
$$

where:

$$
\Gamma(q) \equiv-t e(q) \frac{h^{\prime}(q)}{h(q)}+t h(q)-[e(q)-e(p)] .
$$

Therefore, the SMC is $c+f^{\prime}(g) \Gamma(q)$.

We are now in a position to compare the Pigou effect with the provision effect. Let $s(q)$ stand for the share of consumption in total expenditures on leisure and consumption, i.e., $s(q) \equiv q h(q) / e(q)$. We can show that the following equation and fact are true (Proof in Appendix I.):

$$
\text { Pigou effect }=\sigma \frac{1-s(q)}{s(q)} \times \text { provision effect. }
$$

Fact 3.1. Consider Example 3.2.

(i) If $\sigma \gtreqless 1$, then $s^{\prime}(q) \lesseqgtr 0 \quad \forall q \geq p$.

(ii) If $\sigma>1$, then $\lim _{q \rightarrow \infty} s(q)=0$.

(iii) If $\sigma<1$, then $\lim _{q \rightarrow \infty} s(q)=1$.

(Proof in Appendix I.)

Note that, as pointed out by Chang and Peng (2012, footnote 6), if $\sigma$ exceeds (falls below) unity, then the private goods are gross substitutes (complements). This explains why it matters whether $\sigma$ exceeds unity or not. For example, Eq. (3.24), together with Parts (ii) and (iii) of Fact 3.1, implies that if $\sigma<1$, then the Pigou effect is insignificant, compared with the provision effect, when $q$ is high 
enough. Since Pigou concerns the effect that the commodity tax causes the consumer to substitute the taxed private good by the untaxed private good, it is not surprising that, as indicated by Fact 3.1, if the private goods are gross substitutes (complements), then the Pigou effect is significant (insignificant), compared with the compensated provision effect, if $q$ is high enough.

\section{The SMC Curve}

In Sections 4 and 5, we restrict our attention to Example 3.2. In Section 4, we aim at analyzing the two issues regarding the SMC curve mentioned in the introduction. In other words, we aim at addressing the following two issues: (i) Is the MDL curve upward sloping? (ii) Is the MDL always positive? In Section 4.1, the MDL is regarded as a function of $g$ and $q$, whereas in Section 4.2, we regard the MDL as a function of $g$ by taking into account the fact that $q$ eventually is a function of $g$.

\subsection{The MDL as a Function of Public Good Provision and Post-Tax Price}

We have established that both the Pigou effect and the provision effect are positive, the demand-shift effect is negative, and the provision effect is always dominated by the demand-shift effect. Moreover, if $\sigma \geq 1(\sigma<1)$, then the Pigou effect is significant (insignificant), compared with the provision effect, if $q$ is high enough. These results suggest that if $\sigma \geq 1$ (i.e., the private goods are gross substitutes), then it is possible that the MDL is always positive. In contrast, if $\sigma<1$ (i.e., the private goods are gross complements), then the MDL may be negative. These conjectures are confirmed by the following lemma, which characterizes the $\Gamma(q)$ curve:

Lemma 4.1. Consider Example 3.2.

(i) If $\sigma \geq 1$, then $\Gamma^{\prime}(q)>0 \forall q \geq p$, and hence $\Gamma(q)>0 \forall q \geq p$.

(ii) If $\sigma<1$, then $\Gamma(q)$ is a parabola, i.e., there exists a unique value $q^{M}$ (" $M$ " denotes “maximum") such that $q^{M}$ maximizes $\Gamma(q)$, and $\Gamma^{\prime}(q) \gtreqless 0$, if $q \equiv q^{M}$. Moreover, $q^{M}>2 p$.

(iii) If $\sigma<1$, then $\lim _{q \rightarrow \infty} \Gamma(q)=-\infty$, implying that $\Gamma(q)$ eventually becomes negative, and there exists a unique threshold value $q^{T} \in\left(q^{M}, \infty\right)$ (" $T$ " denotes "threshold") such that $\Gamma(q) \gtreqless 0 \Leftrightarrow q \lesseqgtr q^{T}(q \neq p)$.

(Proof in Appendix I.) 
According to (3.22), the MDL and $\Gamma$ share the same sign. Hence, if $\sigma \geq 1$, then, according to Part (i), the MDL is always positive, and hence, as is established by Wilson (1991a), the level property holds. ${ }^{13}$ In contrast, if $\sigma<1$, then, according to Parts (ii) and (iii), the MDL becomes negative when $q$ is high enough.

Hereafter, we restrict our attention to the case with $\sigma<1$, because if $\sigma \geq 1$, then the sign of the MDL is clear-cut. In order to further explain why the MDL may be negative, let us analyze the commodity tax revenue function:

Fact 4.1. Consider Example 3.2, and assume that $\sigma<1$.

(i) $R_{q}(q, \omega)>0 \quad \forall q \geq p$.

(ii) $R_{q q}(q, \omega)<0 \quad \forall q \geq p$.

(iii) $R(q, \omega)<\omega \quad \forall q \geq p$.

(iv) $\lim _{q \rightarrow \infty} R(q, \omega)=\omega$.

(Proof in Appendix $I^{14}$ )

Part (i) means that higher tax rates can always achieve more tax revenue, i.e., in terms of Chang and Peng (2012, Definition 3.1), the so-called Laffer effect does not exist. ${ }^{15}$ Part (iv) means that the government is able to almost acquire the leisure endowment through taxes on consumption goods, i.e., in terms of Chang and Peng (2012, Definition 3.2), the weak Laffer effect does not exist. ${ }^{16}$

It is interesting to use an argument made by WiLson (1991b) mentioned in Section 3.2, together with Fact 4.1-(iv), to explain why the MDL may be negative. Wilson argues that the total deadweight loss decreases with the size of the distorted private sector. Fact 4.1-(iv) means that if the tax rate is very high, then the distorted private sector is very small, implying that the deadweight loss is very low.

13 According to Lemma 4.1-(i), $\Gamma\left(q^{S}\right)>0$, and hence $f^{\prime}\left(g^{S}\right) \Gamma\left(q^{S}\right)>0$. It follows that $f^{\prime}\left(g^{S}\right) e(p)>c$, which, together with (3.20a), implies that $g^{S}<g^{F}$.

14 According to this proof, when $\sigma=1$, (i)-(iii) are satisfied, whereas (iv) is not.

15 As mentioned previously, the inequality $\sigma \leq 1$ means that the private goods are weak gross complements. Propositions 3.1 and 3.3 of CHANG and Peng (2012) thus imply that the Laffer effect does not exist if and only if $\sigma \leq 1$.

16 In contrast, Chang and Peng (2012, Proposition 3.2) establish that, in general, if the private goods are weak gross substitutes, then the weak Laffer effect exists. 


\subsection{The MDL as a Function of Public Good Provision}

In this subsection, $q$ is regarded as a function of $g$, i.e., $q$ is replaced by $q^{S}(g)$. Fact 4.1 guarantees that $q^{S}(g)$ is well-defined if $0 \leq g<w / c .{ }^{17}$ Equation (3.5) implies that:

$$
\frac{\mathrm{d} q^{S}}{\mathrm{~d} g}=\frac{c}{R_{q}}, \frac{\mathrm{d}^{2} q^{S}}{\mathrm{~d} g^{2}}=-\frac{c R_{q q}}{\left(R_{q}\right)^{2}} \frac{\mathrm{d} q^{S}}{\mathrm{~d} g} .
$$

Accordingly, Parts (i) and (ii) of Fact 4.1 imply that:

$$
\frac{\mathrm{d} q^{S}}{\mathrm{~d} g}>0, \frac{\mathrm{d}^{2} q^{S}}{\mathrm{~d} g^{2}}>0 \quad \forall g \in[0, \omega / c) .
$$

Moreover, Part (iv) implies that:

$$
\lim _{g \rightarrow \omega / c} q^{S}(g)=\infty .
$$

We now characterize the MDL curve, i.e., the $\Phi\left(q^{S}(g), \mathrm{g}\right)$ curve, in the (g,money) space. Note that $\Phi\left(q^{S}(g), g\right)=f^{\prime}(g) \Gamma\left(q^{S}(g)\right)$. We thus begin with studying the $\Gamma\left(q^{S}(g)\right)$ curve. Equations (4.2)-(4.3) imply that there exists a unique $g^{M} \in(0, \omega / c)$ such that $q^{S}\left(g^{M}\right)=q^{M}$. Moreover, there exists a unique $g^{T} \in(0, \omega / c)$ such that $q^{S}\left(g^{T}\right)=q^{T}$. Lemma 4.1 thus leads to the following proposition.

Proposition 4.1. Assume that $\sigma<1$.

(i) $\Gamma\left(q^{S}(g)\right)=0$ if $g=0$.

(ii) The $\Gamma\left(q^{S}(g)\right)$ curve is a parabola, i.e., $\mathrm{d} \Gamma\left(q^{S}(g)\right) / \mathrm{d} g \gtreqless 0 \Leftrightarrow g \lesseqgtr g^{M}$, where $\mathrm{d} \Gamma\left(q^{S}(g)\right) / \mathrm{d} g=\mathrm{d} \Gamma^{\prime}\left(q^{S}(g)\right) \mathrm{d} q^{S} / \mathrm{d} g$. Hence, $g^{M}$ maximizes $\mathrm{d} \Gamma\left(q^{S}(g)\right)$.

(iii) $g^{T} \in\left(g^{M}, \omega / c\right)$.

(iv) If $0<g<\omega / c$, then $\Gamma\left(q^{S}(g)\right) \gtreqless 0 \Leftrightarrow g \lesseqgtr g^{T}$.

(Proof in Appendix I.)

We are in a position to analyze the $f^{\prime}(g) \Gamma\left(q^{S}(g)\right)$ curve in the (g,money) space. As mentioned, the domain of $q^{S}(\cdot)$ is $[0, \omega / c)$. Accordingly, (3.21) implies that

17 Fact 4.1-(i) implies that $q$ and $g$ have a one-to-one relationship, and hence $q^{S}(g)$ is uniquely determined if it exists. Parts (iii) and (iv) of Fact 4.1 imply that range $(R(\cdot, \omega))=[0, \omega)$, and hence the domain of $q^{S}(\cdot)$ is the interval $[0, \omega / c)$, i.e., $q^{S}(g)$ exists if $0 \leq g<\omega / c$. 
$f^{\prime}(g)$ can be regarded as a positive number for each $g$. Parts (i) and (iv) of Proposition 4.1 thus imply:

$$
f^{\prime}(g) \Gamma\left(q^{S}(g)\right) \begin{cases}=0, & \text { if } g=0, \\ >0, & \text { if } 0<g<g^{T}, \\ =0, & \text { if } g=g^{T}, \\ <0, & \text { if } g>g^{T} .\end{cases}
$$

We refer to $\left[0, g^{T}\right]$ as the "normal region," because $g \in\left[0, g^{T}\right] \Rightarrow f^{\prime}(g) \Gamma\left(q^{S}(g)\right) \geq 0$. According to (4.4), if $g$ is high enough, then the MDL is negative. Moreover, we can establish that the $f^{\prime}(g) \Gamma\left(q^{S}(g)\right)$ curve should be upward sloping when $g$ just increases from 0 , whereas it becomes downward sloping if $g$ is high enough (at least when $\left.g \geq g^{M}\right) \cdot{ }^{18}$ In this sense, we say that the MDL curve is at least a "quasiparabola." [As shown in Figures 5.1-5.3, in every one of the three numerical examples considered in Section 5, the MDL curve is actually a parabola.]

We are now in a position to show how to revise the results if (3.21) does not hold.

Remark 4.1. If (3.21) fails, then there is a unique $g^{*} \in(0, \omega / c)$ such that $f^{\prime}\left(g^{*}\right)=0$, implying that the relevant region is $\left[0, g^{*}\right]$, instead of $[0, \omega / c]$. Moreover, from $c>0$, it follows that $g^{F}<g^{*}$. If $g^{*}>g^{T}$, then all the results remain true. When $g^{*} \leq g^{T}$, the result is clear-cut: the $M D L$ is positive in the relevant region, and hence $g^{S}<g^{F}$.

\section{The Three Fundamental Issues}

As mentioned beforehand, in the ( $g$,money) space the MWP curve is downward sloping. Because $c$ is constant, the PMC curve is a horizontal line. Equation (3.16) implies that the MWP curve meets the PMC curve at a unique point, and this intersection point is in the interior. By contrast, according to Proposition 4.1, the SMC curve is initially upward sloping, but becomes downward sloping if $g$ is

18 Let $\Psi(g)$ denote $f^{\prime}(g) \Gamma\left(q^{S}(g)\right)$. It follows that $\Psi^{\prime}(g)=f^{\prime}(g) \mathrm{d} \Gamma\left(q^{S}(g)\right) / \mathrm{d} g+f^{\prime \prime}(g) \Gamma\left(q^{S}(g)\right)$. Parts (i) and (ii) of Proposition 4.1 thus imply that $\Psi^{\prime}(0)>0$. According to $(3.20 \mathrm{a}), f^{\prime \prime}<0$. As a result, if $0<g<g^{T}$, then $\mathrm{d} \Gamma\left(q^{S}(g)\right) / \mathrm{d} g<0 \Rightarrow \Psi^{\prime}(g)<0$. Proposition 4.1 thus implies that $\Psi^{\prime}(g)<0$ $\forall g^{M} \leq g \leq g^{T}$. 
high enough (MDL plus PMC equals SMC). This suggests that the MWP curve may meet the SMC curve at more than one point, and the second-best $g$ may be a corner one. Hence, this section aims at addressing the three fundamental issues mentioned in the introduction. Accordingly, we need to conduct a global analysis, in which we are able to unambiguously depict the whole SMC curve in the ( $g$,money) space, and use this curve to verify the above conjecture. To this end, this section consider three numerical examples of Example 3.2. [We will discuss whether two assumptions in Example 3.2 are restrictive or not after presenting these numerical examples.]

In this section we present three numerical examples of Example 3.2. Each numerical example shares the following assumption:

$$
\omega=5, a=b=c=\frac{1}{2}, p=1 .
$$

Note that if $a=b=1 / 2$ and $p=1$, then $e(p)=4$ (see the equation for $e(q)$ in the proof of Fact 3.1). Moreover, we assume that:

$$
f(g)=\beta_{1} g-\beta_{2} g^{2}
$$

It is clear that $f^{\prime}(g)=\beta_{1}-2 \beta_{2} g$ and $f^{\prime \prime}(g)=-2 \beta_{2}$. Equation(3.16) thus means that:

$$
\begin{gathered}
\beta_{2}>0, \\
0<\beta_{1}-\frac{c}{e(p)}<\frac{2 \beta_{2} \omega}{c} .
\end{gathered}
$$

Hence, $g^{F}$ is in the interior:

$$
0<g^{F}=\frac{\beta_{1}-\frac{c}{e(p)}}{2 \beta_{2}}<\frac{\omega}{c} .
$$

Let us begin by considering the counterexample of GAUBE (2000).

Example 5.1. $\rho=-1, \beta_{1}=3 / 4$, and $\beta_{2}=3 / 80$.

In Figure 5.1, we depict the MWP curve, the PMC curve, and the SMC curve for Example 5.1. The MWP curve and the PMC curve meet at a unique point, which represents the first-best solution, namely, $g^{F}=8.33$, whereas the MWP 
curve and the SMC curve intersect at a unique point, which represents the second-best solution, namely, $g^{S}=8.99$.

Figure 5.1: The Counterexample of GAUBE (2000)

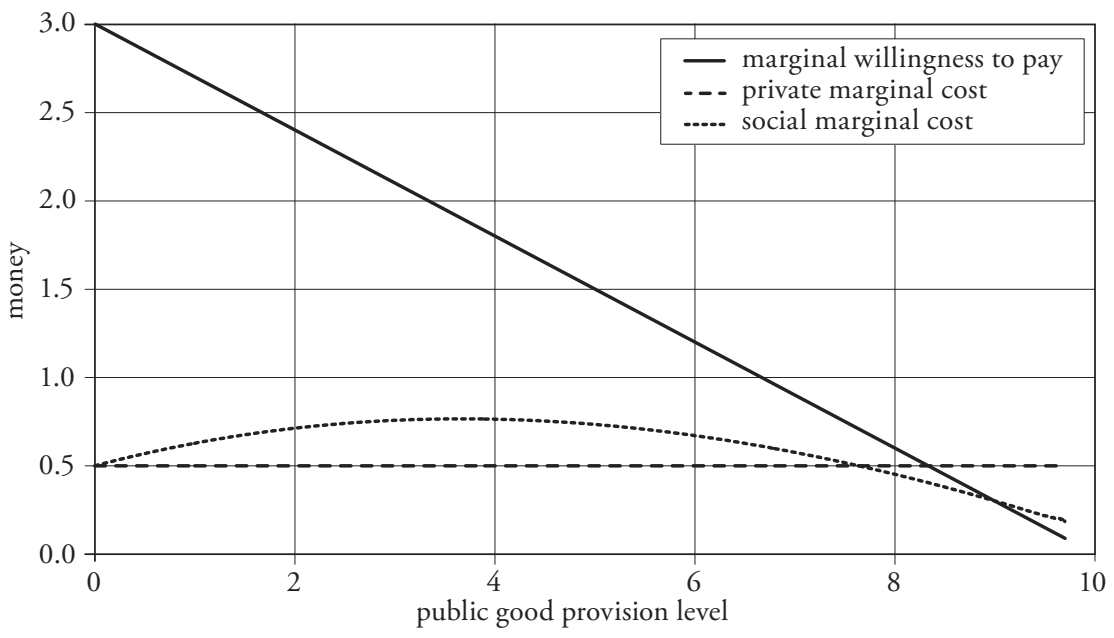

Figure 5.1 suggests that the MWP curve may meet the SMC curve at two points if we shift the MWP curve downward and flatten it (i.e., if either one of $\beta_{1}$ and $\beta_{2}$ is reduced). Accordingly, let us consider the following example.

Example 5.2. $\rho=-1, \beta_{1}=0.15$, and $\beta_{2}=1 / 480$.

In Figure 5.2, we depict the three curves for Example 5.2. The MWP curve and the PMC curve meet at a unique point, which represents the first-best solution, namely, $g^{F}=6.0$. In contrast, the MWP curve and the SMC curve meet at two points: namely, the two intersection points are $g_{L}^{S}=2.462$ and $g_{H}^{S}=7.962$. Note that the MWP curve cuts the SMC curve from above the first time and from below the second time. Therefore, $g_{L}^{S}$ is a local maximum, whereas $g_{H}^{S}$ is a local minimum, implying that $g_{L}^{S}$ is socially better than $g_{H}^{S}$.

As shown in Fact 4.1-(iv), the government is able to almost acquire all the leisure endowment through taxes on consumption goods. Figure 5.2 suggests that it may be socially desirable to let $g$ reach $\omega / c$ as close as possible, because SMC falls below MWP if $g>g_{H}^{S}$. We let $\omega / c-\varepsilon$ represent this corner solution. We can show that if $g=g_{L}^{S}$, then $f(g)=0.3567, q=1.7599$, $e(q)=5.4131$, and $U=0.9237$ 
(the level of private utility). ${ }^{19}$ Therefore, the total utility is 1.2804 . However, if the government devotes all the leisure endowment to the public good, then the total utility is $\mathrm{f}(\omega / c)=1.2917$. This implies that $g_{L}^{S}$ is dominated by $\omega / c-\varepsilon$, and hence $g^{s}$ is $\omega / c-\varepsilon$. [As indicated in Figure 5.2, it seems that the areas of the region lying below the MWP curve and above the SMC curve are larger than the areas of the region lying below the SMC curve and above the MWP curve.]

Figure 5.2: A Case with a Corner Second-Best Solution

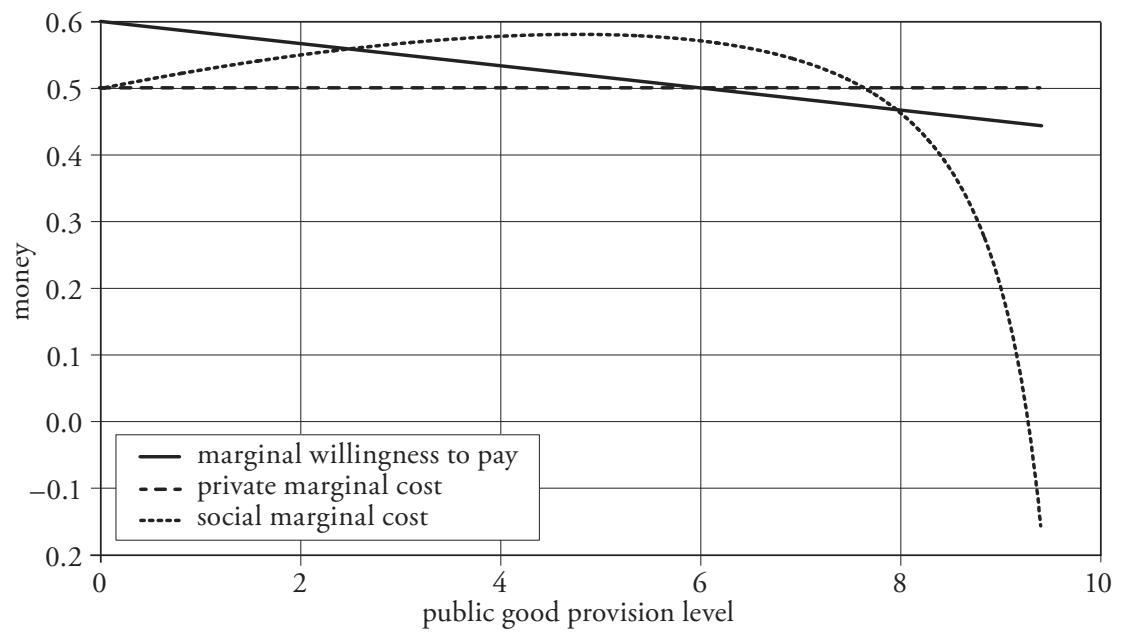

Let us present a case where the smaller interior solution is better than the corner solution. Figure 5.2 suggests that if we shift the MWP curve downward and let it become steeper, then the areas of the region lying below the SMC curve and above the MWP curve become larger and the areas of the region lying below the MWP curve and above the SMC curve become smaller. Therefore, we consider the following example in which $\beta_{1}$ is reduced, whereas $\beta_{2}$ is raised:

Example 5.3. $\rho=-1, \beta_{1}=0.13$, and $\beta_{2}=1 / 240$.

We depict Example 5.3 in Figure 5.3. In this example, the smaller interior solution is $g_{L}^{S}=0.343$. Moreover, we can show that if $g=g_{L}^{S}$, then $f(g)=0.0411$, $q=1.0723, e(q)=4.1433, U=1.2068$, and the total utility is 1.2509 . However, $f(\omega / c)=0.8833$, implying that $g^{S}=g_{L}^{S}$.

19 The level of private utility is equal to $\omega / e(q)$. 
Figure 5.3: A Case with an Interior Second-Best Solution

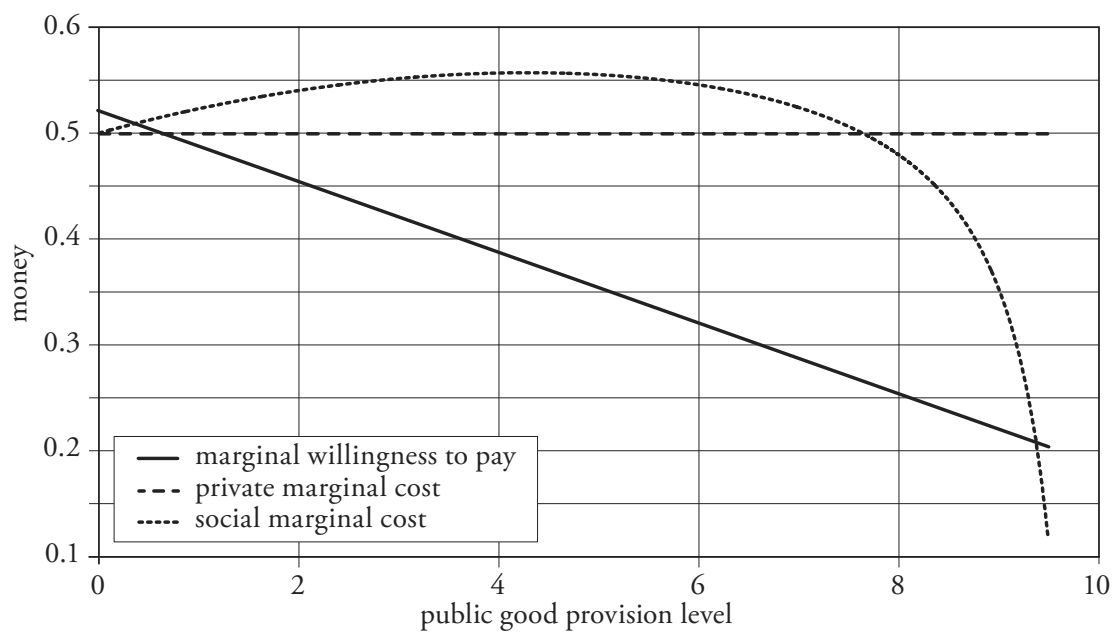

Whether Two Assumptions in Example 3.2 Are Restrictive or not

We are next in a position to discuss the two assumptions regarding the private utility function $U$ in Example 3.2. For the purpose of uncovering a general tendency, it seems reasonable to assume that the elasticity of substitution between $x$ and $\omega+z$ is constant, because the partial derivative of the substitution elasticity with respect to the price of a private good does not have an obvious sign. Note that CES functions are homothetic. Since the issue to be addressed in this paper is an aggregate one, it seems reasonable to assume that $U$ is homothetic with respect to $x$ and $\omega+z$. In contrast, it might be restrictive to assume that $U$ is homogeneous of degree one with respect to $x$ and $\omega+z$. The reason runs as follows. This assumption implies that $W_{u}=0$, and hence, as mentioned in Section 3.1, the income effect vanishes. ${ }^{20}$ If the income effect does not vanish, then it is positive, implying that one may wonder whether it is still possible for the MDL to fall below zero. Accordingly, in Appendix II we relax this assumption by assuming that the total utility function is:

20 WiLson (1991, p. 59) argues that since CES functions are homothetic, we are free to define the total utility function in a way that makes the private utility function $U$ homogeneous of degree 1 with respect to $x$ and $\omega+z$. However, in our view, it seems that the assumption of homogeneous of degree one is not reasonable. 


$$
u=[U(x, \omega+z)]^{n}+f(g),
$$

where $n$ is a positive constant. [In Example 3.2, $n=1$.] We demonstrate that the main result still holds, i.e., the SMC curve is eventually downward sloping and the MDL eventually becomes negative, although the SMC curve is initially upward sloping and the MDL is initially positive.

\section{Conclusions}

We demonstrate in this paper that the SMC curve is well behaved, namely, it is upward sloping everywhere, if and only if the private goods are gross substitutes. Therefore, we next restrict our attention to the gross complementarity case. In this case, there are two unusual properties. First, the SMC curve is initially upward sloping and eventually becomes downward sloping. Second, the SMC curve eventually falls below the PMC curve, i.e., the MDL eventually becomes negative. Since the demand curve is downward sloping, the first property implies that it may meet the SMC curve at two interior points. Moreover, the smaller interior solution is socially better than the larger interior solution, because the former is a local maximum and the latter is a local minimum. The second property implies that it is socially desirable to further raise the level of public good provision if the public good provision level is high enough, implying that there exists a corner solution to the first-order condition of the second-best problem. It is clear that this corner solution is a local maximum. We use a numerical example to establish that it indeed is possible that the smaller interior solution is dominated by the corner solution, and hence the second-best is the corner solution. This result is interesting, because, as mentioned, the first-best solution is assumed to be an interior one.

The two unusual properties remain to be explained. We demonstrate that there are three effects in determining the MDL: the Pigou effect, the provision effect, and the demand-shift effect. [In Example 3.2, the income effect does not exist.] Both the Pigou and provision effects are positive, whereas the demand-shift effect is negative. As a result, the driving force for the MDL to eventually become negative is the demand-shift effect. The demand-shift effect states that an increase in the tax rate will raise the MWP (i.e., marginal willingness to pay) for the public good since it pushes up the unit cost of private utility, hence making the public good be more attractive, relative to the private goods. In other words, the supply of the public good creates its own demand when the funding to cover production costs increases through distortionary commodity taxes! 
An argument made by WiLson (1991b) also can be used to explain why the supply of the public good creates its own demand when the funding to cover production costs increases through distortionary commodity taxes. Wilson argues that a higher public good provision may be viewed as representing "a transfer of resources away from the distorted private sector and into the governmentcontrolled sector. With fewer resources employed in the sector that misallocates them, the deadweight loss should be lower." Hence, a higher public good provision itself reduces the deadweight loss. This argument highlights the role play by the gross complementarity: If the private goods are gross complements, then the government is able to almost acquire the leisure endowment through the commodity tax ${ }^{21}$ i.e., to almost reduce the size of the distorted private sector to be zero.

\section{Appendix I}

The proof of Eq. (3.24). From $s(q)=q h(q) / e(q)$ it follows that:

$$
t h(q)=\frac{t s(q) e(q)}{q}
$$

If $\sigma$ is constant, then, according to (16) of WILson (1991, p. 60),

$$
-h^{\prime}(q)=[1-s(q)] \sigma \frac{h(q)}{q}
$$

implying:

$$
\frac{-t e(q) h^{\prime}(q)}{h(q)}=\sigma \frac{1-s(q)}{s(q)} \frac{t s(q) e(q)}{q}
$$

Comparing the above two equations reveals that Eq. (3.24) is true.

21 It has been argued that it is impossible to tax leisure (including time inputs used in household production). However, the government can implicitly tax leisure by raising taxes on goods that are more complementary to leisure than is the average good, and by lowering taxes on goods that are more substitutable for leisure (CHANg and Peng, 2012, p. 654). 
The proof of Fact 3.1. Solving the expenditure minimization problem (3.9) yields:

$$
\begin{gathered}
h(q)=\frac{1}{a}\left[b+(1-b)\left(\frac{(1-b) q}{b}\right)^{\rho /(1-\rho)}\right]^{-1 / \rho}, \\
z(q)=\frac{\left(\frac{(1-b) q}{b}\right)^{1 /(1-\rho)}}{a}\left[b+(1-b)\left(\frac{(1-b) q}{b}\right)^{\rho /(1-\rho)}\right]^{-1 / \rho}-\omega, \\
s(q)=\frac{q+\left(\frac{(1-b) q}{b}\right)^{1 /(1-\rho)}}{q}, \\
e(q)=q+\frac{\left(\frac{(1-b) q}{b}\right)^{1 /(1-\rho)}}{a}\left[b+(1-b)\left(\frac{(1-b) q}{b}\right)^{\rho /(1-\rho)}\right]^{-1 / \rho} .
\end{gathered}
$$

In order to prove Fact 3.1, we rewrite (A.1c) as the following:

$$
s(q)=\frac{1}{1+\left(\frac{(1-b)}{b}\right)^{\frac{1}{1-\rho}} q^{\frac{1}{1-\rho}-1}} .
$$

Note that $\frac{1}{1-\rho}-1=\sigma-1$. Fact 3.1 thus follows from (A.2). Q.E.D.

The proof of Lemma 4.1. Using (15') of WiLson (1991, p. 60) gives:

$$
\Gamma^{\prime}(q)=-\frac{h^{\prime}(q) q}{s(q)}+t h^{\prime}(q)\left(1+\frac{1}{s(q)}-\sigma\right)
$$


Note that:

$$
-\frac{h^{\prime}(q) q}{s(q)}+\frac{t h^{\prime}(q)}{s(q)}=-\frac{h^{\prime}(q) p}{s(q)}=-\frac{t h^{\prime}(q)}{\tau(q) s(q)}, \forall q>p,
$$

where $\tau$ is the ad valorem tax rate (as a ratio of the producer price):

$$
\tau(q)=\frac{q-p}{p}
$$

Therefore, (A.3) implies:

$$
\Gamma^{\prime}(q)= \begin{cases}-\frac{h^{\prime}(q) q}{s(q)}, & \text { if } q=p, \\ t h^{\prime}(q)\left(1-\sigma-\frac{1}{\tau(q) s(q)}\right), & \text { if } q>p .\end{cases}
$$

According to (A.1a), $h^{\prime}(q)<0 \forall q \geq p$. Equation (A.4) thus implies that $\Gamma^{\prime}(p)>0$. Moreover, if $\sigma \geq 1$, then $\Gamma^{\prime}(q)>0$ for $q>p$. This completes the proof of (i).

We next consider the case with $\sigma<1$. By definition, $\tau(p) s(p)=0$, and $\tau(q)$ is an increasing function. According to Fact 3.1-(i), $s^{\prime}(q)>0 \forall q \geq p$. Moreover, according to Fact 3.1-(iii), $\lim _{q \rightarrow \infty} s(q)=1$. Therefore, $\lim _{q \rightarrow \infty} \tau(q) s(q)=\infty$. There thus exists a unique $q^{M}>p$ such that $\tau\left(q^{M}\right) s\left(q^{M}\right)=1 /(1-\sigma)$ and:

$$
\Gamma^{\prime}(q) \gtreqless 0 \Leftrightarrow q \lesseqgtr q^{M},
$$

where it is assumed that $q \neq p$. There are two implications. First, $q^{M}$ maximizes $\Gamma(q)$. Second, $\tau\left(q^{M}\right)=1 /\left[(1-\sigma) s\left(q^{M}\right)\right]$, which is higher than unity. Therefore, $q^{M}>2 p$. This completes the proof of (ii).

Fact 3.1-(iii), together with (3.24), implies that when $q$ is high enough, compared to the compensated provision effect, the Pigou effect can be neglected. Therefore:

$$
\lim _{q \rightarrow \infty} \Gamma(q)=\lim _{q \rightarrow \infty}\{t h(q)-[e(q)-e(p)]\} .
$$

From the facts that $t h(q)=(q-p) h(q)$ and $e(q)=q h(q)+\omega+z(q)$, it follows that $t h(q)-[e(q)-e(p)]=-p h(q)-(\omega+z(q))+e(p)$. 
The inequality $\sigma<1$ means that $\rho<0$. From $\rho<0$, it follows that $\rho /$ $(1-\rho)<0$, implying:

$$
\lim _{q \rightarrow \infty}\left(\frac{(1-b) q}{b}\right)^{\rho /(1-\rho)}=0
$$

This equation, together with (A.1a), (A.1b) and (A.1d), implies that:

$$
\begin{gathered}
\lim _{q \rightarrow \infty} h(q)=\frac{b^{-1 / \rho}}{a}, \\
\lim _{q \rightarrow \infty} z(q)=\lim _{q \rightarrow \infty} \frac{b^{-1 / \rho}}{a} \times\left(\frac{1-b}{b}\right)^{1 /(1-\rho)} \times q^{1 /(1-\rho)}, \\
\lim _{q \rightarrow \infty} e(q)=\frac{b^{-1 / \rho}}{a} \times q .
\end{gathered}
$$

It thus follows that:

$$
\lim _{q \rightarrow \infty} \Gamma(q)=\lim _{q \rightarrow \infty}-z(q)
$$

namely, $\lim _{q \rightarrow \infty} \Gamma(q)=-\infty$, which, together with (A.5), implies that there exists a unique $q^{T}$ such that $\Gamma\left(q^{T}\right)=0$. Accordingly, from $\Gamma(p)=0$ and (A.5) it follows that $\Gamma(q)>0 \forall p \in\left(\mathrm{p}, q^{T}\right), \Gamma\left(q^{T}\right)=0$, and $\Gamma(q)<0 \forall q>q^{T}$. This completes the proof of (iii). Q.E.D.

The proof of Fact 4.1. Solving the first-order condition for the problem $\max _{x, z} U(x, \omega+z)$ s.t. $q x+(\omega+z)=\omega+y$ yields:

$$
\begin{aligned}
& X(q, \omega+y)=\frac{\omega+y}{q+\left(\frac{(1-b) q}{b}\right)^{1 /(1-\rho)}}, \\
& R(q, \omega+y)=\frac{(q-p)(\omega+y)}{q+\left(\frac{(1-b) q}{b}\right)^{1 /(1-\rho)} .} .
\end{aligned}
$$


Equation (A.8a) implies that the share of $x$ in total expenditure on the private goods only depends upon $q$, and hence we can write $s(q)$ for $q X(q, \omega+y) /(\omega+y)$ :

$$
s(q)=\frac{q}{q+\left(\frac{(1-b) q}{b}\right)^{1 /(1-\rho)} .}
$$

This is the same as (A.1c). According to (18) of WILsON (1991, p. 60):

$$
\frac{d \ln s}{d \ln q}=(1-s)(1-\sigma) .
$$

We write $\varepsilon$ for $-\partial \ln X / \partial \ln q$. By the definition of $s, X=s \times(\omega+y) / q$. From (A.9), it thus follows that $\varepsilon$ only depends upon $q$ :

$$
\varepsilon(q)=1-[1-s(q)](1-\sigma)=\sigma+s(q)(1-\sigma) .
$$

It is known that:

$$
R_{q}=X(q, \omega+y)[1-\hat{\tau}(q) \varepsilon(q)],
$$

where $\hat{\tau}(q)$ stands for the tax rate (as a ratio of the consumer price): $\hat{\tau}(q) \equiv(q-p) / q$. From the definition of $\hat{\tau}(q)$, it follows that:

$$
\begin{aligned}
& \hat{\tau}(p)=0 ; \\
& 0<\hat{\tau}(q)<1, \quad \forall q>p ; \\
& \hat{\tau}^{\prime}(q)>0, \quad \forall q \geq p ; \\
& \lim _{q \rightarrow+\infty} \hat{\tau}(q)=1 .
\end{aligned}
$$

Equation (A.11) reveals that it is important to compare $\hat{\tau}$ with $1 / \varepsilon$ in order to determine the sign of $R_{q}$ :

$$
R_{q} \gtreqless 0 \Leftrightarrow \hat{\tau} \lesseqgtr \frac{1}{\varepsilon} .
$$

Equation (A.10) guarantees that if $\sigma \leq 1$, then $1 / \varepsilon(q) \geq 1 \forall q \geq p$, which, together with (A.12), implies that $\hat{\tau}(q)<1 / \varepsilon(q) \forall q \geq p$. [Note that $\sigma$ is allowed to be unity.] Hence, $R_{q}$ is always strictly positive. This completes the proof of (i). 
Equation (A.11), together with (A.9) and (A.10), implies:

$$
R_{q q}=-\frac{X}{q^{2}}\{(1-\sigma)(1-s)[\sigma+2 s(1-\sigma)] t+2 p[1-(1-\sigma)(1-s)]\}
$$

If $0<\sigma \leq 1$, then $\{\cdot\}>0$, implying that $R_{q q}$ is strictly negative for all $q \geq p$. [Note that $\sigma$ is allowed to be unity.] This completes the proof of (ii).

Part (iii) is trivial. Note that $\sigma<1 \Rightarrow 1 /(1-\rho)<1$. Part (iv) thus follows from (A.8b). [Note that $\sigma$ is not allowed to be unity.] Q.E.D.

The proof of Proposition 4.1. Equation (3.23) implies that $\Gamma(p)=0$, and hence Part (i) follows from the fact that $q^{S}(0)=p$. According to (4.1), $\mathrm{d} q^{S} / \mathrm{d} g$ is strictly positive. This has three implications. First, Parts (iii) and (iv) straightforwardly follow from Lemma 4.1-(iii). Second, $\mathrm{d} \Gamma\left(q^{S}(g)\right) / \mathrm{d} g$ and $\Gamma^{\prime}\left(q^{S}(g)\right)$ share the same sign. Third, Lemma 4.1-(ii) implies that $\Gamma^{\prime}\left(q^{S}(g)\right) \gtreqless 0 \Leftrightarrow g \equiv g^{M}$. Part (ii) follows from the latter two implications. Q.E.D.

\section{Appendix II}

Consider the case in which the total utility function is (5.6). It is clear that the two Marshallian functions $X(q, \omega+y)$ and $R(q, \omega+y)$ do not change, i.e., (3.12) and (3.13) still hold. Moreover, the post-tax price function $q^{S}(g)$ remains the same. However, the indirect utility function depends on $n$ :

$$
V(q, g, \omega+y)=\left(\frac{\omega+y}{e(q)}\right)^{n}+f(g) .
$$

It follows that:

$$
E(q, g, u)=(u-f(g))^{\frac{1}{n}} e(q)
$$

It is known that $H=E_{q}$ and $W=-E_{g}$. It follows that:

$$
\begin{gathered}
H(q, g, u)=(u-f(g))^{\frac{1}{n}} h(q), \\
W(q, g, u)=(u-f(g))^{\frac{1}{n}-1} \frac{f^{\prime}(g) e(q)}{n} .
\end{gathered}
$$


Equation (A.15) implies that:

$$
V_{y y} \lesseqgtr 0, \text { if } n \lesseqgtr 1 .
$$

Therefore, it seems not reasonable to assume that $n>1$. Moreover, we have examined the case with $n=1$. Accordingly, hereafter it is assumed that $n<1$. Equation (A.17) implies that:

$$
W \gtreqless 0 \text {, if } n \lesseqgtr 1 \text {. }
$$

Therefore, the public good is strictly normal.

Consider the first-best regime. Equation (A.17) implies that (3.1) can be written as:

$$
(u-f(g))^{\frac{1}{n}-1} \frac{f^{\prime}(g) e(p)}{n}=c .
$$

Since $u=V(p, g, \omega-c g), g^{F}$ is determined by:

$$
\frac{1}{n} \times\left(\frac{\omega-c g}{e(p)}\right)^{1-n} \times f^{\prime}(g) e(p)=c .
$$

Consider the second-best regime. Equations (A.16)-(A.17) imply that the Pigou effect is represented by

$$
\left(\frac{[u-f(g)]^{\frac{1}{n}-1}}{n}\right) \times\left(\frac{-t e(q) h^{\prime}(q) f^{\prime}(g)}{h(q)}\right)>0 ;
$$

the compensated provision effect is

$$
\left(\frac{[u-f(g)]^{\frac{1}{n}-1}}{n}\right) \times\left[t h(q) f^{\prime}(g)\right]>0 ;
$$


the demand-shift effect is

$$
\left(\frac{[u-f(g)]^{\frac{1}{n}-1}}{n}\right) \times\left\{-[e(q)-e(p)] f^{\prime}(g)\right\}<0 .
$$

It follows that:

$$
\Phi(q, g, u)=\left(\frac{[u-f(g)]^{\frac{1}{n}-1}}{n}\right) \times f^{\prime}(g) \Gamma(q) .
$$

From $u=V(q, g, \omega)$ it follows that:

$$
[u-f(g)]^{\frac{1}{n}-1}=\left(\frac{\omega}{e(q)}\right)^{1-n} .
$$

Therefore, $g^{S}$ is determined by the following equation:

$$
\frac{1}{n} \times\left(\frac{\omega}{e(q)}\right)^{1-n} \times f^{\prime}(g) e(p)=c+\frac{1}{n} \times\left(\frac{\omega}{e(q)}\right)^{1-n} \times f^{\prime}(g) \Gamma(q) .
$$

We can rewrite (A.18) as:

$$
\begin{aligned}
\frac{1}{n} \times\left(\frac{\omega-c g}{e(p)}\right)^{1-n} f^{\prime}(g) e(p) & =c+\frac{1}{n} \times\left[\left(\frac{\omega-c g}{e(p)}\right)^{1-n}-\left(\frac{\omega}{e(q)}\right)^{1-n}\right] \\
& \times f^{\prime}(g) e(p)+\frac{1}{n} \times\left(\frac{\omega}{e(q)}\right)^{1-n} \\
& \times f^{\prime}(g) \Gamma(q),
\end{aligned}
$$

where the second term of the right-hand side represents the income effect, and the third term represent the three other effects.

We are in a position to study the four effects. Since the public good is strictly normal, the income effect is positive everywhere. We next study the following three effects: Pigou, compensated provision, and demand-shift effects. Note that these three effects share the same multiplier. We can draw three implications 
from this fact. First, the compensated provision effect is still dominated by the demand-shift effect. Second, Eq. (3.24) still holds. Third, the counterpart of Eq. (4.4) still holds:

$$
\frac{1}{n} \times\left(\frac{\omega}{e\left(q^{S}(g)\right)}\right)^{1-n} \times f^{\prime}(g) \Gamma\left(q^{S}(g)\right) \begin{cases}=0, & \text { if } g=0, \\ >0, & \text { if } 0<g<g^{T}, \\ =0, & \text { if } g=g^{T}, \\ <0, & \text { if } g>g^{T} .\end{cases}
$$

Equation (A.19), together with the fact that the income effect is positive, implies that the MDL is positive when $g \in\left(0, g^{T}\right]$. It follows that the SMC curve is initially upward sloping, because the MDL is zero when $g=0$.

We next analyze whether it is possible for the MDL to be negative. From $n-1>0$ it follows that:

$$
\lim _{g \rightarrow \omega / c}[\omega-\operatorname{cge}(p)]^{1-n} \times f^{\prime}(g) e(p)=0 .
$$

From:

$$
0<[\omega-\operatorname{cge}(p)]^{1-n}-[\omega e(q)]^{1-n}<[\omega-\operatorname{cge}(p)]^{1-n},
$$

it thus follows that the income effect eventually becomes negligible:

$$
\lim _{g \rightarrow \omega / c}\left[\left(\frac{\omega-c g}{e(p)}\right)^{1-n}-\left(\frac{\omega}{e(q)}\right)^{1-n}\right] \times f^{\prime}(g) e(p)=0 .
$$

Equations (4.1)-(4.3) imply that:

$$
\lim _{g \rightarrow \omega / c}\left(\frac{\omega}{e\left(q^{S}(g)\right)}\right)^{1-n} f^{\prime}(g) \Gamma\left(q^{S}(g)\right)=\omega^{1-n} f^{\prime}\left(\frac{\omega}{c}\right) \times \lim _{q \rightarrow \infty} \frac{\Gamma(q)}{[e(q)]^{1-n}} .
$$

Equations (A.6b), (A.6c), and (A.7) in Appendix I imply that:

$$
\lim _{q \rightarrow \infty} \frac{\Gamma(q)}{[e(q)]^{1-n}}=-\lim _{q \rightarrow \infty}\left(\frac{b^{-1 / \rho}}{a}\right)^{n} \times\left(\frac{1-b}{b}\right)^{\sigma} \times \frac{q^{\sigma}}{q^{1-n}} .
$$


It follows that:

$$
\begin{aligned}
\lim _{g \rightarrow \omega / c}\left[\omega e\left(q^{S}(g)\right)\right]^{1-n} f^{\prime}(g) \Gamma\left(q^{S}(g)\right) & \begin{array}{ll}
-\infty, & \text { if } n>1-\sigma, \\
-\omega^{1-n} f^{\prime}\left(\frac{\omega}{c}\right) \times\left(\frac{b^{-1 / \rho}}{a}\right)^{n} \times\left(\frac{1-b}{b}\right)^{\sigma}, & \text { if } n=1-\sigma, \\
0, & \text { if } n<1-\sigma .
\end{array}
\end{aligned}
$$

Equations (A.20)-(A.21) imply that if $\mathrm{n} \geq 1-\sigma$, then we have a clear-cut result: When $g$ is high enough, the income effect is dominated by the other three effects taken together (i.e., the sum of the Pigou, income, and compensated provision effects is dominated by the demand-shift effect) and hence the MDL is negative, implying that the SMC curve is eventually downward sloping.

\section{References}

Aronsson, Thomas (2008), "Social Accounting and the Public Sector", International Economic Review, 49 (1), pp. 349-75.

Atrinson, Anthony B., and Nicholas H. Stern (1974), "Pigou, Taxation and Public Goods", Review of Economic Studies, 41, pp. 119-28.

Atrinson, Anthony B., and Joseph E. Stiglitz (1980), Lectures on Public Economics, McGraw-Hill, New York.

Ballard, Charles L. and Don Fullerton (1992), "Distortionary Taxes and the Provision of Public Goods", Journal of Economic Perspective, 6 (3) pp. 117-31.

Batina, Raymond G. (1990), "On the Interpretation of the Modified Samuelson Rule for Public Goods in Static Models with Heterogeneity," Journal of Public Economics, 42, pp. 125-33.

Batina, Raymond G., and Toshiniro Ihori (2005), Public Goods: Theories and Evidence, New York: Springer.

Browning, Edgar K., Timothy Gronberg and Liqun Liu (2000), "Alternative Measures of the Marginal Cost of Funds," Economic Inquiry, 38 (4), pp. 591-9. Chang, Ming C.(2000), "Rules and Levels in the Provision of Public Goods: the Role of Complementarities between the Public Good and Taxed Commodities," International Tax and Public Finance, 7, pp. 83-91. 
Chang, Ming C., and Hsiao-Ping Peng(2009), "Laffer Effect, Gross Substitution, Marginal Cost of Public Funds and the Level Property of Public Good Provision," International Tax and Public Finance, 19 (5), pp.650-9.

Christiansen, Vidar (2007), "Two Approaches to Determine Public Good Provision under Distortionary Taxation," National Tax Journal, 2007, 60 (1), 25-43.

Dahlby, Bev (2008), The Marginal Cost of Public Funds: Theory and Applications, MIT Press, Cambridge, Mass. and London.

de Bartolome, Charles A.M. (1996), "Is Pigou Wrong? Can Distortionary Taxation Cause Public Spending to Exceed the Efficient Level?” unpublished mimeo.

de Bartolome, Charles A.M. (1997), "Slow Adjustment and the Level of Government Spending," Journal of Urban Economics, 42 (2), pp. 285-311.

Diamond, Peter A. (1975), "A Many-Person Ramsey Rule", Journal of Public Economics, 4, pp. 335-42.

Diamond, Peter A., and James A. Mirrlees (1971), "Optimal Taxation and Public Production I: Production Efficiency," American Economic Review, 61, pp. 261-78.

Gaube, Thomas (2000), "When do Distortionary Taxes Reduce the Optimal Supply of Public Goods?” Journal of Public Economics, 76 (2), pp. 151-80.

Gronberg, Timothy, and Liqun Liu (2001), "The Second-Best Level of a Public Good: An Approach Based on the Marginal Excess Burden," Journal of Public Economic Theory, 3 (4), pp.431-53.

King, Mervyn A. (1986), "A Pigovian Rule for the Optimum Provision of Public Goods", Journal of Public Economics, 30, pp. 273-91.

NG, Yew-Kwang (2000), "The Optimal size of Public Spending and the Distortionary Cost of Taxation”, National Tax Journal, 53 (2), pp. 253-72.

Pigou, Arthur C. (1947), Public Finance, Macmillan, London.

Slemrod, Joel, and Shlomo Yitzhaki (2001), "Integrating Expenditure and Tax Decisions: The Marginal Cost of Funds and the Marginal Benefit of Projects," National Tax Journal, 54 (2), pp. 189-201.

Stern, Nicholas (1986), "A Note on Commodity Taxation: The Choice of Variable and the Slutsky, Hessian and Antonelli Matrices (SHAM)", Review of Economic Studies, 53, pp. 293-9.

Triest, Robert K. (1990), "The Relationship between the Marginal Cost of Public Funds and Marginal Excess Burden," The American Economic Review, 80 (3), pp. 557-66.

Wilson, John D. (1991), "Optimal Public Good Provision in the Ramsey Tax Model - A Generalization,” Economics Letters, 35, pp. 57-61. 
Wilson, John D. (1991), "Optimal Public Good Provision with Limited LumpSum Taxation," American Economic Review, 81 (1), pp. 153-66. 